\title{
ON SINGULARITIES OF HAMILTONIAN MAPPINGS
}

\author{
TAKUO FUKUDA \\ Department of Mathematics \\ College of Humanities and Sciences, Nihon University \\ 3-25-40 Sakurajousui, Setagaya-ku, Tokyo, Japan \\ STANISŁAW JANECZKO \\ Institute of Mathematics, Polish Academy of Sciences \\ Sniadeckich 8, Warszawa, Poland, and \\ Faculty of Mathematics and Information Science, Warsaw University of Technology \\ Plac Politechniki 1, 00-661 Warszawa, Poland \\ E-mail: janeczko@impan.gov.pl
}

\begin{abstract}
The notion of an implicit Hamiltonian system-an isotropic mapping $H: M \rightarrow$ $(T M, \dot{\omega})$ into the tangent bundle endowed with the symplectic structure defined by canonical morphism between tangent and cotangent bundles of $M$-is studied. The corank one singularities of such systems are classified. Their transversality conditions in the 1-jet space of isotropic mappings are described and the corresponding symplectically invariant algebras of Hamiltonian generating functions are calculated.
\end{abstract}

1. Introduction. Let $(M, \omega)$ be a symplectic manifold. A Hamiltonian system is an isotropic section $F: M \rightarrow T M$ of the tangent bundle $T M$ endowed with the symplectic structure defined by the canonical morphism $\beta$ between tangent and cotangent bundles of $M$ appearing in the commuting diagram (cf. [16])

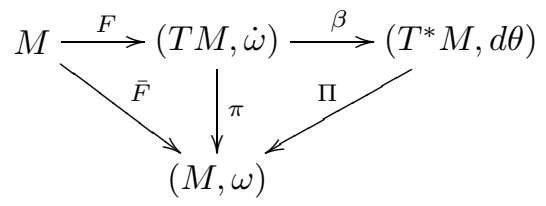

We have $F^{*} \dot{\omega}=0$ and $\bar{F}=\pi \circ F, \dot{\omega}=\beta^{-1}(d \theta)$.

2000 Mathematics Subject Classification: 37J35, 57R45, 58C35, 70H05.

Key words and phrases: Hamiltonian system, isotropic mapping, singularity, implicit system.

The work was supported by EC FP6 MC ToK programmes TODEQ, MTKD-CT-2005030042 and SPADE2, MTKD-CT-2004-014508.

The paper is in final form and no version of it will be published elsewhere. 
If we consider $F$ to be a smooth isotropic regular mapping of another manifold $N$ $(\operatorname{dim} N=\operatorname{dim} M)$ into $T M$ then $F$ is a parametrization of a Hamiltonian system in $(T M, \dot{\omega})$, which in general if $\bar{F}$ has singularities (cf. [12]) is an implicit Hamiltonian system (see e.g. $[1,2,5,6,10,11,15]$ ). For each isotropic mapping $F$ there exists at least locally a generating Hamiltonian function $h: N \rightarrow \mathbb{R}$ such that $(\beta \circ F)^{*} \theta=-d h$. In this paper we study the symplectically invariant algebra of Hamiltonian generating functions determined by the mapping $\bar{F}$ or more precisely by its singularity type. In the corank one singularity case this algebra is defined by the ideal generated by the determinant of the Jacobi matrix of $\bar{F}$. The algebra of generating Hamiltonian functions in the general corank case singularity of $\bar{F}$ is calculated and conditions on an isotropic map $F$ ensuring that the one-jet extension $j^{1} F$ is transversal to the corank one stratum in the isotropic 1-jet space of mappings are derived. These conditions are obtained if, first, $\bar{F}$ has corank one singularity and then corank $k$ singularity with $k \geq 2$.

2. Isotropic mappings. Let $\left(\mathbb{R}^{2 n}, \omega\right)$ be a Euclidean symplectic space, $\omega=\sum_{i=1}^{n} d y_{i} \wedge$ $d x_{i}$ in canonical Darboux coordinates $(x, y)=\left(x_{1}, \ldots, x_{n}, y_{1}, \ldots, y_{n}\right)$.

Let $\theta$ be the Liouville 1 -form on the cotangent bundle $T^{*} \mathbb{R}^{2 n}$. Then $d \theta$ is the standard symplectic structure on $T^{*} \mathbb{R}^{2 n}$. Let $\beta: T \mathbb{R}^{2 n} \rightarrow T^{*} \mathbb{R}^{2 n}$ be the canonical bundle map defined by $\omega$,

$$
\beta: T \mathbb{R}^{2 n} \ni v \mapsto \omega(v, \cdot) \in T^{*} \mathbb{R}^{2 n} .
$$

Then we can define the canonical symplectic structure $\dot{\omega}$ on $T \mathbb{R}^{2 n}$,

$$
\dot{\omega}=\beta^{*} d \theta=d\left(\beta^{*} \theta\right)=\sum_{i=1}^{n}\left(d \dot{y}_{i} \wedge d x_{i}-d \dot{x}_{i} \wedge d y_{i}\right),
$$

where $(x, y, \dot{x}, \dot{y})$ are local coordinates on $T \mathbb{R}^{2 n}$ and $\beta^{*} \theta=\sum_{i=1}^{n}\left(\dot{y}_{i} d x_{i}-\dot{x}_{i} d y_{i}\right)$.

Throughout the paper if not otherwise stated all objects are germs at 0 of smooth functions, mappings, forms etc. or their representatives on an open neighbourhood of 0 in $\mathbb{R}^{2 n}$.

Definition 2.1. Let $F:\left(\mathbb{R}^{2 n}, 0\right) \rightarrow T \mathbb{R}^{2 n}$ be a smooth map-germ. We say that $F$ is isotropic if $F^{*} \dot{\omega}=0$.

Proposition 2.2. A smooth map-germ $F:\left(\mathbb{R}^{2 n}, 0\right) \rightarrow T \mathbb{R}^{2 n}$ is isotropic if and only if there exists a smooth function-germ $h:\left(\mathbb{R}^{2 n}, 0\right) \rightarrow \mathbb{R}$ such that

$$
(\beta \circ F)^{*} \theta=-d h \text {. }
$$

For each smooth isotropic map-germ $F$ such a function-germ $h$ is unique up to an additive constant.

Proof. We assume that $F:\left(\mathbb{R}^{2 n}, 0\right) \rightarrow T \mathbb{R}^{2 n}$ is isotropic, then the differential of the 1 -form $(\beta \circ F)^{*} \theta$ defined on some contractible open neighbourhood $U \subset \mathbb{R}^{2 n}$ of 0 vanishes,

$$
d(\beta \circ F)^{*} \theta=F^{*} \beta^{*} d \theta=F^{*} \dot{\omega}=0 .
$$

Thus $(\beta \circ F)^{*} \theta$ is a closed 1-form on $U$. By the Poincaré Lemma, there exists a smooth function $h: \mathbb{R}^{2 n} \supset U \rightarrow \mathbb{R}$ such that $(\beta \circ F)^{*} \theta=-d h$. For each smooth isotropic map- 
germ $F:\left(\mathbb{R}^{2 n}, 0\right) \rightarrow T \mathbb{R}^{2 n}$ there exists a unique (up to an additive constant) smooth function-germ $h:\left(\mathbb{R}^{2 n}, 0\right) \rightarrow \mathbb{R}$ such that $(2.1)$ is fulfilled.

Let $(u, v)=\left(u_{1}, \ldots, u_{n}, v_{1}, \ldots, v_{n}\right)$ denote coordinates of the source space $U \subset \mathbb{R}^{2 n}$. In local coordinates we define $F=(f, g, \dot{f}, \dot{g}): \mathbb{R}^{2 n} \supset U \rightarrow T \mathbb{R}^{2 n}$, and $\bar{F}=\pi \circ F=$ $(f, g): \mathbb{R}^{2 n} \supset U \rightarrow \mathbb{R}^{2 n}$, where $\pi$ denotes the canonical projection $\pi: T \mathbb{R}^{2 n} \rightarrow \mathbb{R}^{2 n}$. By

$$
J(\bar{F})=\left(\begin{array}{ll}
\frac{\partial f}{\partial u} & \frac{\partial f}{\partial v} \\
\frac{\partial g}{\partial u} & \frac{\partial g}{\partial v}
\end{array}\right)
$$

we denote the Jacobi matrix of $\bar{F}$, i.e. the matrix of the tangent map $d \bar{F}$, and by $I_{n}$ the unit matrix of dimension $n$.

Lemma 2.3. A smooth map-germ $F:\left(\mathbb{R}^{2 n}, 0\right) \rightarrow T \mathbb{R}^{2 n}$ is isotropic if and only if there exists a smooth function-germ $h:\left(\mathbb{R}^{2 n}, 0\right) \rightarrow \mathbb{R}$ such that

$$
\left(\begin{array}{c}
\frac{\partial h}{\partial u} \\
\frac{\partial h}{\partial v}
\end{array}\right)={ }^{t}\left(\begin{array}{ll}
\frac{\partial f}{\partial u} & \frac{\partial f}{\partial v} \\
\frac{\partial g}{\partial u} & \frac{\partial g}{\partial v}
\end{array}\right)\left(\begin{array}{cc}
O & -I_{n} \\
I_{n} & O
\end{array}\right)\left(\begin{array}{l}
\dot{f} \\
\dot{g}
\end{array}\right) .
$$

Proof. Equivalence of (2.1) and (2.2) can be verified by comparing $(\beta \circ F)^{*} \theta\left(\frac{\partial}{\partial u_{i}}\right)$ and $(\beta \circ F)^{*} \theta\left(\frac{\partial}{\partial v_{i}}\right)$ with $d h\left(\frac{\partial}{\partial u_{i}}\right)=\frac{\partial h}{\partial u_{i}}$ and $d h\left(\frac{\partial}{\partial v_{i}}\right)=\frac{\partial h}{\partial v_{i}}$ respectively. For we get

$$
\begin{aligned}
(\beta \circ F)^{*} \theta\left(\frac{\partial}{\partial u_{i}}\right) & =F^{*} \beta^{*} \theta\left(\frac{\partial}{\partial u_{i}}\right)=F^{*}\left(\sum_{j=1}^{n} \dot{y}_{j} d x_{j}-\dot{x}_{j} d y_{j}\right)\left(\frac{\partial}{\partial u_{i}}\right) \\
& =\left(\sum_{j=1}^{n} \dot{g}_{j} d x_{j}-\dot{f}_{j} d y_{j}\right)\left(d F\left(\frac{\partial}{\partial u_{i}}\right)\right)=\sum_{j=1}^{n}\left(\frac{\partial f_{j}}{\partial u_{i}} \dot{g}_{j}-\frac{\partial g_{j}}{\partial u_{i}} \dot{f}_{j}\right)
\end{aligned}
$$

and in the same way

$$
(\beta \circ F)^{*} \theta\left(\frac{\partial}{\partial v_{i}}\right)=\sum_{j=1}^{n}\left(\frac{\partial f_{j}}{\partial v_{i}} \dot{g}_{j}-\frac{\partial g_{j}}{\partial v_{i}} \dot{f}_{j}\right) .
$$

In general $F$ can be regarded as a vector field along $\bar{F}$, i.e. a section of the induced

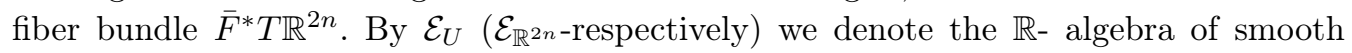
function germs at 0 on $U \subset \mathbb{R}^{2 n}$ (and on "the target space" $\mathbb{R}^{2 n}$ respectively). From Proposition 2.2, for each isotropic map-germ $F$ along $\bar{F}$ there exists a unique $h$ belonging to the maximal ideal $\mathbf{m}_{U}$ of $\mathcal{E}_{U}$, which we call a generating function-germ for $F$.

Let $F:(U, 0) \rightarrow T \mathbb{R}^{2 n}$ and $G:(U, 0) \rightarrow T \mathbb{R}^{2 n}$ be two isotropic map-germs along $\bar{F}:(U, 0) \rightarrow \mathbb{R}^{2 n}$ and $\bar{G}:(U, 0) \rightarrow \mathbb{R}^{2 n}$ respectively. Now we introduce the natural equivalence groups acting on isotropic mappings through a natural lifting of diffeomorphic or symplectomorphic equivalences of $\bar{F}$ and $\bar{G}$ (cf. $[8,9]$ ).

Definition 2.4. 1. Let $F:(U, 0) \rightarrow T \mathbb{R}^{2 n}$ and $G:(U, 0) \rightarrow T \mathbb{R}^{2 n}$ be two isotropic mapgerms. We say that $F$ and $G$ are Lagrangian equivalent if there exist a diffeomorphismgerm $\varphi:(U, 0) \rightarrow(U, 0)$, and a symplectomorphism-germ $\Psi:\left(T \mathbb{R}^{2 n}, 0\right) \rightarrow\left(T \mathbb{R}^{2 n}, 0\right)$, $\Psi^{*} \dot{\omega}=\dot{\omega}$, preserving the fibering $\pi$ such that $G=\Psi \circ F \circ \varphi$. 
2. Let $F:(U, 0) \rightarrow T \mathbb{R}^{2 n}$ and $G:(U, 0) \rightarrow T \mathbb{R}^{2 n}$ be two isotropic map-germs along $\bar{F}:(U, 0) \rightarrow \mathbb{R}^{2 n}$ and $\bar{G}:(U, 0) \rightarrow \mathbb{R}^{2 n}$ respectively. We say that $F$ and $G$ are $L$ symplectic equivalent if there exist a diffeomorphism-germ $\varphi:(U, 0) \rightarrow(U, 0)$, and a symplectomorphism-germ $\Psi:\left(T \mathbb{R}^{2 n}, 0\right) \rightarrow\left(T \mathbb{R}^{2 n}, 0\right), \Psi^{*} \dot{\omega}=\dot{\omega}$, preserving the fibering $\pi$ and a symplectomorphism-germ $\Phi:\left(\mathbb{R}^{2 n}, 0\right) \rightarrow\left(\mathbb{R}^{2 n}, 0\right), \Phi^{*} \omega=\omega, \pi \circ \Psi=\Phi \circ \pi$, such that $G=\Psi \circ F \circ \varphi$ and $\bar{G}=\Phi \circ \bar{F} \circ \varphi$. In this case we call $\bar{F}$ and $\bar{G}$ symplectomorphic or symplectically equivalent.

To $\bar{F}$ we associate the symplectically invariant algebra $\mathcal{R}_{\bar{F}}$ of all generating functiongerms (cf. [8]),

$$
\begin{aligned}
\mathcal{R}_{\bar{F}} & =\left\{h \in \mathcal{E}_{U}: h \text { generates an isotropic map-germ along } \bar{F}\right\} \\
& =\left\{h \in \mathcal{E}_{U}: d h \in \mathcal{E}_{U} d\left(\bar{F}^{*} \mathcal{E}_{\mathbb{R}^{2 n}}\right)\right\} .
\end{aligned}
$$

It is easy to check that if $\bar{F}$ has a maximal rank then $\mathcal{R}_{\bar{F}}=\mathcal{E}_{U}$. The aim of this section is to study the case when $\bar{F}$ has no maximal rank and establish the structure of $\mathcal{R}_{\bar{F}}$.

Now we assume that $\bar{F}$ is a corank one map-germ at $0 \in U$. Let $e \in T_{0} U$ span the kernel of the Jacobi matrix $J(\bar{F})$ at zero. By $\Delta_{\bar{F}}$ we denote the determinant of $J(\bar{F})$ and by $\partial_{e}$ the derivation in $e$-direction.

TheOREm 2.5. Let $F:\left(\mathbb{R}^{2 n}, 0\right) \rightarrow T \mathbb{R}^{2 n}$ be a smooth map-germ such that $\bar{F}$ has corank one singularity at 0 .

1. If $F$ is isotropic then there exists a unique generating function-germ $h:\left(\mathbb{R}^{2 n}, 0\right) \rightarrow$ $\mathbb{R}, h(0)=0$ such that $\partial_{e} h \in\left\langle\Delta_{\bar{F}}\right\rangle$, where $\left\langle\Delta_{\bar{F}}\right\rangle$ is the ideal generated by $\Delta_{\bar{F}}$ in $\mathcal{E}_{\mathbb{R}^{2 n}}$.

2. Conversely, for every smooth function-germ $h:\left(\mathbb{R}^{2 n}, 0\right) \rightarrow \mathbb{R}$ such that $\partial_{e} h \in\left\langle\Delta_{\bar{F}}\right\rangle$ there is a unique isotropic map-germ $F:\left(\mathbb{R}^{2 n}, 0\right) \rightarrow T \mathbb{R}^{2 n}$ such that $\bar{F}=\pi \circ F$ and $(\beta \circ F)^{*} \theta=-d h$.

Proof. Since we have assumed that the corank of $\bar{F}=(f, g):(U, 0) \rightarrow \mathbb{R}^{2 n}$ is one at the origin, we can choose coordinates in $U$ such that

$$
\begin{aligned}
& f_{i}(u, v)=u_{i}, \quad i=1, \ldots, n, \\
& g_{i}(u, v)=v_{i}, \quad i=1, \ldots, n-1, \\
& \frac{\partial g_{n}}{\partial v_{n}}(0,0)=0
\end{aligned}
$$

and $e=\frac{\partial}{\partial v_{n}}$. Then

$$
J(\bar{F})=\left(\begin{array}{ccc}
I_{n} & O & 0 \\
O & I_{n-1} & 0 \\
\frac{\partial g_{n}}{\partial u} & \frac{\partial g_{n}}{\partial \bar{v}} & \frac{\partial g_{n}}{\partial v_{n}}
\end{array}\right)
$$

where $\bar{v}=\left(v_{1}, \ldots, v_{n-1}\right)$. 
Since $\dot{f}, \dot{g}$ in the equation (2.2) are smooth, we can write equivalently

$$
\begin{aligned}
\left(\begin{array}{c}
\dot{f} \\
\dot{g}
\end{array}\right) & =\left(\begin{array}{cc}
O & I_{n} \\
-I_{n} & O
\end{array}\right)^{t}\left(\begin{array}{cc}
\frac{\partial f}{\partial u} & \frac{\partial f}{\partial v} \\
\frac{\partial g}{\partial u} & \frac{\partial g}{\partial v}
\end{array}\right)^{-1}\left(\begin{array}{c}
\frac{\partial h}{\partial u} \\
\frac{\partial h}{\partial v}
\end{array}\right) \\
& =\left(\begin{array}{cc}
O & I_{n} \\
-I_{n} & O
\end{array}\right)\left(\begin{array}{ccc}
I_{n} & O & -\frac{\partial g_{n}}{\partial u} / \Delta_{\bar{F}} \\
O & I_{n-1} & -\frac{\partial g_{n}}{\partial \bar{v}} / \Delta_{\bar{F}} \\
0 & 0 & 1 / \Delta_{\bar{F}}
\end{array}\right)\left(\begin{array}{c}
\frac{\partial h}{\partial u} \\
\frac{\partial h}{\partial v}
\end{array}\right) \\
& =\left(\begin{array}{cc}
O & I_{n} \\
-I_{n} & O
\end{array}\right)\left(\begin{array}{c}
\frac{\partial h}{\partial u}-\frac{\partial g_{n}}{\partial u} \frac{\partial h}{\partial v_{n}} / \Delta_{\bar{F}} \\
\frac{\partial h}{\partial \bar{v}}-\frac{\partial g_{n}}{\partial \bar{v}} \frac{\partial h}{\partial v_{n}} / \Delta_{\bar{F}} \\
\frac{\partial h}{\partial v_{n}} / \Delta_{\bar{F}}
\end{array}\right) .
\end{aligned}
$$

Thus in order that the right hand side of (2.4) should be smooth we get

$$
\frac{\partial h}{\partial v_{n}} \in\left\langle\Delta_{\bar{F}}\right\rangle
$$

so we proved item 1 . If we have $h$ which fulfills the condition $(2.5)$ then by the formula

$$
\left(\begin{array}{c}
\dot{f} \\
\dot{g}
\end{array}\right)=\left(\begin{array}{cc}
O & I_{n} \\
-I_{n} & O
\end{array}\right)^{t}\left(\begin{array}{cc}
\frac{\partial f}{\partial u} & \frac{\partial f}{\partial v} \\
\frac{\partial g}{\partial u} & \frac{\partial g}{\partial v}
\end{array}\right)^{-1}\left(\begin{array}{c}
\frac{\partial h}{\partial u} \\
\frac{\partial h}{\partial v}
\end{array}\right)
$$

we construct $F$ in a unique way.

COROLlaRY 2.6. The algebra $\mathcal{R}_{\bar{F}}$ of all generating function-germs (which is also an

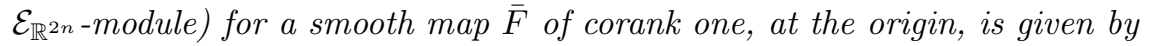

$$
\mathcal{R}_{\bar{F}}=\left\{h \in \mathcal{E}_{U}: \partial_{e} h \in\left\langle\Delta_{\bar{F}}\right\rangle\right\}
$$

If $\bar{F}:(U, 0) \rightarrow\left(\mathbb{R}^{2 n}, 0\right)$ is a corank $1 C^{\infty}$ stable map-germ, then by B. Morin's classification theorem [14] $\bar{F}$ is diffeomorphic to one of the following so-called $A_{k}$ type singularities, $0<k<2 n$ :

$$
\left(u_{1}, \ldots, u_{2 n}\right) \mapsto\left(u_{1}, \ldots, u_{2 n-1}, u_{2 n}^{k+1}+\sum_{i=1}^{k-1} u_{i} u_{2 n}^{k-i}\right)
$$

We call a $C^{\infty}$ stable map-germ diffeomorphic to the normal form of $A_{k}$ type singularity also an $A_{k}$ type singularity. In this note, we classify corank $1 C^{\infty}$ stable map-germs $\bar{F}:(U, 0) \rightarrow\left(\mathbb{R}^{2 n}, 0\right)$ under the symplectomorphic equivalence.

TheOREM 2.7. Let $\bar{F}:(U, 0) \rightarrow\left(\mathbb{R}^{2 n}, 0\right)$ be an $A_{k}$ type singularity. Then $\bar{F}$ is symplectomorphic to the following map-germ:

$$
u=\left(u_{1}, \ldots, u_{2 n}\right) \mapsto\left(u_{1}, \ldots, u_{2 n-1}, u_{2 n}^{k+1}+\sum_{i=1}^{k-1} a_{i}(u) u_{2 n}^{k-i}\right),
$$

where $a_{1}(u), \ldots, a_{k-1}(u)$ are smooth function-germs such that $d a_{1}, \ldots, d a_{k-1}$ and $d u_{2 n}$ are linearly independent at the origin.

Proof. Let $\bar{F}:(U, 0) \rightarrow\left(\mathbb{R}^{2 n}, 0\right)$ be an $A_{k}$ type singularity. Let $\left(w_{1}, \ldots, w_{2 n}\right)$ denote the standard coordinates in $U$. Then there exist diffeomorphism-germs $\phi=\left(\phi_{1}, \ldots, \phi_{2 n}\right)$ : 


$$
\begin{gathered}
(U, 0) \rightarrow(U, 0) \text { and } \psi=\left(\psi_{1}, \ldots, \psi_{2 n}\right):\left(\mathbb{R}^{2 n}, 0\right) \rightarrow\left(\mathbb{R}^{2 n}, 0\right) \text { such that } \\
\psi_{i} \circ \bar{F} \circ \phi\left(w_{1}, \ldots, w_{2 n}\right)=w_{i}, \quad i=1,2, \ldots, 2 n-1, \\
\psi_{2 n} \circ \bar{F} \circ \phi\left(w_{1}, \ldots, w_{2 n}\right)=w_{2 n}^{k+1}+\sum_{i=1}^{k-1} w_{i} w_{2 n}^{k-i} .
\end{gathered}
$$

Since $d \psi_{2 n}$ does not vanish at the origin, there exists a symplectic coordinate system $\left(\varphi_{1}, \ldots, \varphi_{2 n}=\psi_{2 n}\right)$ with $\varphi_{2 n}=\psi_{2 n}$. Set

$$
\begin{aligned}
u_{i} & =\varphi_{i} \circ \bar{F} \circ \phi\left(w_{1}, \ldots, w_{2 n}\right), \quad i=1,2, \ldots, 2 n-1, \\
u_{2 n} & =w_{2 n} .
\end{aligned}
$$

Note that the map-germ $\Phi=\left(\left(\varphi_{1}, \ldots, \varphi_{2 n}\right):\left(\mathbb{R}^{2 n}, 0\right) \rightarrow\left(\mathbb{R}^{2 n}, 0\right)\right.$ is a symplectomorphism.

Now we show that $\left(u_{1}, \ldots, u_{2 n}\right)$ is a new coordinate system in $(U, 0)$. In fact for functions $\alpha_{1}, \ldots, \alpha_{k}$ and variables $w_{1}, \ldots, w_{m}$, let us denote the Jacobian matrix at the origin of $\alpha_{1}, \ldots, \alpha_{k}$ with respect to $w_{1}, \ldots, w_{m}$ by

$$
J\left(\frac{\alpha_{1}, \ldots, \alpha_{k}}{w_{1}, \ldots, w_{m}}\right)(0) .
$$

Then, since both of $\psi=\left(\psi_{1}, \ldots, \psi_{2 n}\right)$ and $\left(\varphi_{1}, \ldots, \varphi_{2 n}\right)$ are local coordinate systems in the target space, we have

$$
\begin{aligned}
& \operatorname{rank} J\left(\frac{\varphi_{1} \circ \bar{F} \circ \phi, \ldots, \varphi_{2 n} \circ \bar{F} \circ \phi}{w_{1}, \ldots, w_{2 n-1}}\right)(0) \\
& =\operatorname{rank} J\left(\frac{\psi_{1} \circ \bar{F} \circ \phi, \ldots, \psi_{2 n} \circ \bar{F} \circ \phi}{w_{1}, \ldots, w_{2 n-1}}\right)(0)=2 n-1 .
\end{aligned}
$$

Since $\varphi_{2 n}=\psi_{2 n}$ and since

$$
J\left(\frac{\varphi_{2 n} \circ \bar{F} \circ \phi}{w_{1}, \ldots, w_{2 n-1}}\right)(0)=J\left(\frac{\psi_{2 n} \circ \bar{F} \circ \phi}{w_{1}, \ldots, w_{2 n-1}}\right)(0)=(0, \ldots, 0),
$$

we have

$$
\begin{aligned}
& \operatorname{rank} J\left(\frac{u_{1}, \ldots, u_{2 n-1}}{w_{1}, \ldots, w_{2 n-1}}\right)(0) \\
& =\operatorname{rank} J\left(\frac{\varphi_{1} \circ \bar{F} \circ \phi, \ldots, \varphi_{2 n-1} \circ \bar{F} \circ \phi}{w_{1}, \ldots, w_{2 n-1}}\right)(0)=2 n-1 .
\end{aligned}
$$

Thus $\left(u_{1}, \ldots, u_{2 n-1}, u_{2 n}=w_{2 n}\right)$ is a coordinate system.

Now, from (2.8) and (2.9), we have

$$
\begin{aligned}
\varphi_{i} \circ \bar{F} \circ \phi & =u_{i}, \quad i=1,2, \ldots, 2 n-1, \\
\varphi_{2 n} \circ \bar{F} \circ \phi & =u_{2 n}^{k+1}+\sum_{i=1}^{k-1} w_{i} u_{2 n}^{k-i} .
\end{aligned}
$$

Setting $a_{i}(u)=w_{i}$, we obtain (2.7). This completes the proof of Theorem 2.7.

COROLlaRY 2.8 (Symplectomorphic normal form of folds). Let $\bar{F}:(U, 0) \rightarrow\left(\mathbb{R}^{2 n}, 0\right)$ be a fold singularity, i.e. an $A_{1}$ type singularity. Then $\bar{F}$ is symplectomorphic to the normal 
form of the fold:

$$
\left(u_{1}, \ldots, u_{2 n}\right) \mapsto\left(u_{1}, \ldots, u_{2 n-1}, u_{2 n}^{2}\right) .
$$

Thus the fold type singularities have only one symplectomorphic type.

For $\bar{F}=(f, g)$ with corank $J(\bar{F})(0,0)=k \geq 2$ we get that $\bar{F}$ can be reduced by symplectomorphic equivalence to the form

$$
\begin{aligned}
f_{i}(u, v) & =u_{i}, & & i=1, \ldots, n-k_{1}, \\
g_{i}(u, v) & =v_{i}, & & i=1, \ldots, n-k_{2}, \\
\frac{\partial f_{i}}{\partial u_{j}}(0,0) & =0, & & n-k_{1}<i, j \leq n, \\
\frac{\partial f_{i}}{\partial v_{j}}(0,0) & =0, & & n-k_{1}<i \leq n, n-k_{2}<j \leq n, k_{1} \geq k_{2}, \\
\frac{\partial g_{i}}{\partial u_{j}}(0,0) & =0, & & n-k_{2}<i \leq n, n-k_{1}<j \leq n, \\
\frac{\partial g_{i}}{\partial v_{j}}(0,0) & =0, & & n-k_{2}<i, j \leq n,
\end{aligned}
$$

i.e. up to a smooth coordinate change in $U$ and a symplectomorphic coordinate transformation of $\left(\mathbb{R}^{2 n}, \omega\right)$.

Below we will consider the special but representative case when $\bar{F}$ can be reduced by symplectomorphic equivalence to the form

$$
\begin{aligned}
f_{i}(u, v) & =u_{i}, & i & =1, \ldots, n, \\
g_{i}(u, v) & =v_{i}, & i & =1, \ldots, n-k, \\
\frac{\partial g_{i}}{\partial v_{j}}(0,0) & =0, & n-k & <i, j \leq n .
\end{aligned}
$$

with $k^{2} \leq 2 n$. The corresponding Jacobi matrix of $\bar{F}$ can be written in the form

$$
\begin{aligned}
\left(\begin{array}{cc}
\frac{\partial f}{\partial u} & \frac{\partial f}{\partial v} \\
\frac{\partial g}{\partial u} & \frac{\partial g}{\partial v}
\end{array}\right)= & \left(\begin{array}{ccc}
I_{n} & O & O \\
O & I_{n-k} & O \\
\frac{\partial g_{i}}{\partial u_{j}} & \frac{\partial g_{i}}{\partial v_{\ell}} & \frac{\partial g_{i}}{\partial v_{m}}
\end{array}\right), \\
& n-k<i \leq n, 1 \leq j \leq n, \\
& 1 \leq \ell \leq n-k<m \leq n .
\end{aligned}
$$

and in blocks

$$
\left(\begin{array}{ll}
\frac{\partial f}{\partial u} & \frac{\partial f}{\partial v} \\
\frac{\partial g}{\partial u} & \frac{\partial g}{\partial v}
\end{array}\right)=\left(\begin{array}{ccc}
I_{n} & O & O \\
O & I_{n-k} & O \\
C & D_{1} & D_{2}
\end{array}\right)
$$

In this case we can prove a more general version of Theorem 2.5.

THEOREM 2.9. Let $F=(f, g, \dot{f}, \dot{g}):\left(\mathbb{R}^{2 n}, 0\right) \rightarrow T \mathbb{R}^{2 n}$ be a smooth map-germ such that $\operatorname{corank} J(\bar{F})(0,0)=k \geq 2$ and $\bar{F}$ has the form (2.13). Then $F$ is isotropic if and only if there exists a smooth function $h$ on $U$ such that

$$
\left(\begin{array}{c}
\frac{\partial h}{\partial u} \\
\frac{\partial h}{\partial v}
\end{array}\right)={ }^{t}\left(\begin{array}{ll}
\frac{\partial f}{\partial u} & \frac{\partial f}{\partial v} \\
\frac{\partial g}{\partial u} & \frac{\partial g}{\partial v}
\end{array}\right)\left(\begin{array}{cc}
O & -I_{n} \\
I_{n} & O
\end{array}\right)\left(\begin{array}{l}
\dot{f} \\
\dot{g}
\end{array}\right)
$$


which is equivalent to the condition that the component functions of the $k$-vector given by

$$
\left(\begin{array}{c}
\frac{\partial h}{\partial v_{n-k+1}} \\
\frac{\partial}{\frac{\partial h}{\partial v_{n}}}
\end{array}\right)={ }^{t} \tilde{D}_{2}\left(\begin{array}{c}
\frac{\partial h}{\partial v_{n-k+1}} \\
\vdots \\
\frac{\partial h}{\partial v_{n}}
\end{array}\right)
$$

belong to $\langle\operatorname{det} J(f, g)\rangle=\left\langle\Delta_{\bar{F}}\right\rangle$,

$$
\frac{\widetilde{\partial h}}{\partial v_{i}} \in\left\langle\operatorname{det} D_{2}\right\rangle=\langle\operatorname{det} J(f, g)\rangle=\left\langle\Delta_{\bar{F}}\right\rangle, \quad n-k<i \leq n,
$$

where $\tilde{D}_{2}$ is the cofactor matrix of $D_{2}$.

Proof. By matrix calculations we have

$$
\begin{aligned}
\left(\begin{array}{c}
\dot{f} \\
\dot{g}
\end{array}\right) & =\left(\begin{array}{cc}
O & I_{n} \\
-I_{n} & O
\end{array}\right)^{t}\left(\begin{array}{ccc}
I_{n} & O & O \\
O & I_{n-k} & O \\
C & D_{1} & D_{2}
\end{array}\right)^{-1}\left(\begin{array}{c}
\frac{\partial h}{\partial u} \\
\frac{\partial h}{\partial v}
\end{array}\right) \\
& =\left(\begin{array}{ccc}
O_{(n-k) \times n} & I_{n-k} & -{ }^{t} D_{1}{ }^{t} D_{2}^{-1} \\
O_{k \times n} & O_{k \times(n-k)} & { }^{t} D_{2}^{-1} \\
-I_{n} & O_{n \times(n-k)} & { }^{t} C^{t} D_{2}^{-1}
\end{array}\right)\left(\begin{array}{c}
\frac{\partial h}{\partial u} \\
\frac{\partial h}{\partial v}
\end{array}\right) .
\end{aligned}
$$

Thus we have

$$
\begin{aligned}
\left(\begin{array}{c}
\dot{f}_{1} \\
\vdots \\
\dot{f}_{n-k}
\end{array}\right) & =\left(\begin{array}{c}
\frac{\partial h}{\partial v_{1}} \\
\vdots \\
\frac{\partial h}{\partial v_{n-k}}
\end{array}\right)-{ }^{t} D_{1}{ }^{t} D_{2}^{-1}\left(\begin{array}{c}
\frac{\partial h}{\partial v_{n-k+1}} \\
\vdots \\
\frac{\partial h}{\partial v_{n}}
\end{array}\right), \\
\left(\begin{array}{c}
\dot{f}_{n-k+1} \\
\vdots \\
\dot{f}_{n}
\end{array}\right) & ={ }^{t} D_{2}^{-1}\left(\begin{array}{c}
\frac{\partial h}{\partial v_{n-k+1}} \\
\vdots \\
\frac{\partial h}{\partial v_{n}}
\end{array}\right), \\
\left(\begin{array}{c}
\dot{g}_{1} \\
\vdots \\
\dot{g}_{n}
\end{array}\right) & =-\left(\begin{array}{c}
\frac{\partial h}{\partial u_{1}} \\
\vdots \\
\frac{\partial h}{\partial u_{n}}
\end{array}\right)+{ }^{t} C^{t} D_{2}^{-1}\left(\begin{array}{c}
\frac{\partial h}{\partial v_{n-k+1}} \\
\vdots \\
\frac{\partial h}{\partial v_{n}}
\end{array}\right)
\end{aligned}
$$

Since the map $(\dot{f}, \dot{g})$ is smooth, all the functions on the right-hand side must be smooth, which holds if and only if

$$
{ }^{t} D_{2}^{-1}\left(\begin{array}{c}
\frac{\partial h}{\partial v_{n-k+1}} \\
\vdots \\
\frac{\partial h}{\partial v_{n}}
\end{array}\right)={ }^{t}\left(\begin{array}{ccc}
\frac{\partial g_{n-k+1}}{\partial v_{n-k+1}} & \cdots & \frac{\partial g_{n-k+1}}{\partial v_{n}} \\
\cdots & \cdots & \cdots \\
\frac{\partial g_{n}}{\partial v_{n-k+1}} & \cdots & \frac{\partial g_{n}}{\partial v_{n}}
\end{array}\right)^{-1}\left(\begin{array}{c}
\frac{\partial h}{\partial v_{n-k+1}} \\
\vdots \\
\frac{\partial h}{\partial v_{n}}
\end{array}\right)
$$

is smooth.

For a square matrix $A$ of size $k$, let $\widetilde{A}$ denote the cofactor matrix of $A$, i.e. the matrix whose $(i, j)$ entry is the cofactor of the $(j, i)$ entry of $A$. Then we have

$$
\widetilde{A} A=A \widetilde{A}=\operatorname{det} A I_{k} .
$$


Then the formula $(2.23)$ is equal to

$$
\frac{1}{\operatorname{det} D_{2}}{ }^{t} \widetilde{D}_{2}\left(\begin{array}{c}
\frac{\partial h}{\partial v_{n-k+1}} \\
\vdots \\
\frac{\partial h}{\partial v_{n}}
\end{array}\right) .
$$

Let us denote the $i-n+k$-th component of ${ }^{t} \widetilde{D}^{t}\left(\frac{\partial h}{\partial v_{n-k+1}}, \ldots, \frac{\partial h}{\partial v_{n}}\right)$ by $\frac{\widetilde{\partial h}}{\partial v_{i}}$ (for $n-k+1 \leq$ $i \leq n)$,

$$
{ }^{t} \widetilde{D}_{2}\left(\begin{array}{c}
\frac{\partial h}{\partial v_{n-k+1}} \\
\vdots \\
\frac{\partial h}{\partial v_{n}}
\end{array}\right)=\left(\begin{array}{c}
\frac{\partial h}{\partial v_{n-k+1}} \\
\vdots \\
\frac{\partial h}{\partial v_{n}}
\end{array}\right) .
$$

Thus the right-hand side of (2.19), as a result of (2.20)-(2.22), is smooth if and only if

$$
\frac{\widetilde{\partial h}}{\partial v_{i}} \in\left\langle\operatorname{det} D_{2}\right\rangle=\langle\operatorname{det} J(f, g)\rangle=\left\langle\Delta_{\bar{F}}\right\rangle, \quad n-k<i \leq n \text {. }
$$

3. Transversality of isotropic mappings. We find a condition on an isotropic mapgerm $F$ ensuring that $j^{1} F$ is transversal to the corank 1 stratum in the isotropic 1 -jet space of mappings when $\bar{F}$ has corank 1 at the origin. The case of corank $k$ singularity of $\bar{F}$ when $k>2$ will also be considered. But the case $k=2$ we leave to the forthcoming paper.

Let us identify the space of 1 -jets $J^{1}\left(\mathbb{R}^{2 n}, T \mathbb{R}^{2 n}\right)$ with $\mathbb{R}^{2 n} \times T \mathbb{R}^{2 n} \times M(4 n, 2 n)$, where $M(4 n, 2 n)$ is the set of $4 n \times 2 n$ matrices,

$$
J^{1}\left(\mathbb{R}^{2 n}, T \mathbb{R}^{2 n}\right)=\mathbb{R}^{2 n} \times T \mathbb{R}^{2 n} \times M(4 n, 2 n)=\mathbb{R}^{2 n} \times T \mathbb{R}^{2 n} \times \mathbb{R}^{2 n \times 4 n} .
$$

Let $(a, b, c, d)=\left(a_{1}, \ldots, a_{n}, b_{1}, \ldots, b_{n}, c_{1}, \ldots, c_{n}, d_{1}, \ldots, d_{n}\right)$ denote the canonical coordinates of $\mathbb{R}^{4 n}=T \mathbb{R}^{2 n}$ endowed with the symplectic structure

$$
\dot{\omega}=\sum_{i=1}^{n}\left(d d_{i} \wedge d a_{i}-d c_{i} \wedge d b_{i}\right)
$$

Let $A={ }^{t}\left(a_{i j}, b_{i j}, c_{i j}, d_{i j}\right) \in M(4 n, 2 n), 1 \leq i \leq n, 1 \leq j \leq 2 n$ and ${ }^{t}\left(\boldsymbol{a}_{j}, \boldsymbol{b}_{j}, \boldsymbol{c}_{j}, \boldsymbol{d}_{j}\right)$ denotes the $j$-th column of $A, 1 \leq j \leq 2 n$. Then

$$
A \text { is isotropic if } \dot{\omega}\left({ }^{t}\left(\boldsymbol{a}_{i}, \boldsymbol{b}_{i}, \boldsymbol{c}_{i}, \boldsymbol{d}_{i}\right),{ }^{t}\left(\boldsymbol{a}_{j}, \boldsymbol{b}_{j}, \boldsymbol{c}_{j}, \boldsymbol{d}_{j}\right)\right)=0,
$$

for all $1 \leq i, j \leq 2 n$, where

$$
\dot{\omega}\left({ }^{t}\left(\boldsymbol{a}_{i}, \boldsymbol{b}_{i}, \boldsymbol{c}_{i}, \boldsymbol{d}_{i}\right),{ }^{t}\left(\boldsymbol{a}_{j}, \boldsymbol{b}_{j}, \boldsymbol{c}_{j}, \boldsymbol{d}_{j}\right)\right):=\sum_{k=1}^{n}\left(\left(a_{k j} d_{k i}-a_{k i} d_{k j}\right)-\left(b_{k j} c_{k i}-b_{k i} c_{k j}\right)\right) .
$$

We define the subsets

$$
\begin{gathered}
I=I(4 n, 2 n)=\{A \in M(4 n, 2 n): A \text { is isotropic }\}, \\
I_{k}=I_{k}(4 n, 2 n)=\{A \in I(4 n, 2 n): \operatorname{corank} A=k\} .
\end{gathered}
$$

By $\overline{I_{k}}$ we denote the topological closure of $I_{k}$,

$$
\overline{I_{k}}=I_{k} \cup I_{k+1} \cup \cdots \cup I_{2 n} .
$$


$I(4 n, 2 n)$ has singularities along $\overline{I_{2}}$ and $I(4 n, 2 n)-\overline{I_{2}}$ is a codimension $n(2 n-1)$ smooth submanifold of $M(2 n, 4 n)$. Let $J_{I}^{1}\left(\mathbb{R}^{2 n}, T \mathbb{R}^{2 n}\right)$ denote the space of 1 -jets of isotropic maps with corank at most 1 , i.e.

$$
J_{I}^{1}\left(\mathbb{R}^{2 n}, T \mathbb{R}^{2 n}\right)=\mathbb{R}^{2 n} \times T \mathbb{R}^{2 n} \times\left(I(4 n, 2 n)-\overline{I_{2}}\right) .
$$

Let $F: \mathbb{R}^{2 n} \supset U \rightarrow T \mathbb{R}^{2 n}$ be a smooth isotropic map such that $F$ and $\bar{F}=\pi \circ F$ have a corank 1 singularity at $(0,0) \in \mathbb{R}^{2 n}$ and $\bar{F}$ has the form (2.3) in local coordinates $(u, v)$. Consider the 1 -jet extension $j^{1} F: U \rightarrow J_{I}^{1}\left(\mathbb{R}^{2 n}, T \mathbb{R}^{2 n}\right)$ given by $j^{1} F(u, v)=$ $(u, v, F(u, v), J(F)(u, v))$. Thus, $j^{1} F$ is transversal to the corank 1 stratum $\mathbb{R}^{2 n} \times T \mathbb{R}^{2 n} \times$ $I_{1}$ if and only if $J(F): U \rightarrow I(4 n, 2 n)-\overline{I_{2}}$ is transversal to $I_{1}$. Now we seek a genericity condition for $F$ in order that $J(F)$ be transversal to $I_{1}$.

If $F$ is isotropic, then by Theorem 2.5 for its generating function $h$ we have

$$
\frac{\partial h}{\partial v_{n}}(u, v)=\alpha(u, v) \Delta_{\bar{F}}
$$

for some smooth function $\alpha(u, v)$.

Proposition 3.1. The corank of $J(F)(u, v)$ is 1 if and only if

$$
\Delta_{\bar{F}}(u, v)=0 \quad \text { and } \quad \frac{\partial \alpha}{\partial v_{n}}(u, v)=0 .
$$

Proof. For the purpose of this proof we use the notation $\left(w_{1}, \ldots, w_{2 n}\right)=\left(u_{1}, \ldots, u_{n}\right.$, $\left.v_{1}, \ldots, v_{n}\right)$. Since the corank of $J(\bar{F})(0,0)$ is 1 , corank $J(F)(u, v) \leq 1$. Thus in order that $\operatorname{corank} J(F)(u, v)=1$, it is necessary that $\Delta_{\bar{F}}(u, v)=0$. So, under the assumption that $\Delta_{\bar{F}}(u, v)=0$, we prove that the corank of $J(F)(u, v)$ is one if and only if $\frac{\partial \alpha}{\partial w_{2 n}}(u, v)=0$.

If corank $J(F)(u, v)=1$ then we have

$$
0=\frac{\partial \dot{f}_{n}}{\partial w_{2 n}}(u, v)=\frac{\partial \alpha}{\partial w_{2 n}}(u, v) .
$$

Thus we have $\frac{\partial \alpha}{\partial w_{2 n}}(u, v)=0$.

Now suppose that $\frac{\partial \alpha}{\partial w_{2 n}}(u, v)=0$. From (3.1) we can write

$$
\begin{aligned}
\frac{\partial \dot{f}_{i}}{\partial w_{j}} & =\frac{\partial^{2} h}{\partial w_{n+i} \partial w_{j}}-\frac{\partial^{2} g_{n}}{\partial w_{n+i} \partial w_{j}} \alpha-\frac{\partial g_{n}}{\partial w_{n+i}} \frac{\partial \alpha}{\partial w_{j}}, \\
\frac{\partial \dot{f}_{n}}{\partial w_{j}} & =\frac{\partial \alpha}{\partial w_{j}}, \\
\frac{\partial \dot{g}_{i}}{\partial w_{j}} & =-\frac{\partial^{2} h}{\partial w_{i} \partial w_{j}}+\frac{\partial^{2} g_{n}}{\partial w_{i} \partial w_{j}} \alpha+\frac{\partial g_{n}}{\partial w_{i}} \frac{\partial \alpha}{\partial w_{j}}, \quad j=1, \ldots, 2 n .
\end{aligned}
$$

Thus the $j$-th column $\mathbf{a}_{j}$ of the jacobian matrix $J(F)$ for $j<2 n$ can be written in the form

$$
\begin{array}{r}
\mathbf{a}_{j}={ }^{t}\left(0, \ldots, 1,0, \ldots, 0, \frac{\partial g_{n}}{\partial w_{j}}, \ldots, \frac{\partial^{2} h}{\partial w_{n+i} \partial w_{j}}-\frac{\partial^{2} g_{n}}{\partial w_{n+i} \partial w_{j}} \alpha-\frac{\partial g_{n}}{\partial w_{n+i}} \frac{\partial \alpha}{\partial w_{j}}\right. \\
\left.\ldots, \frac{\partial \alpha}{\partial w_{j}}, \ldots,-\frac{\partial^{2} h}{\partial w_{i} \partial w_{j}}+\frac{\partial^{2} g_{n}}{\partial w_{i} \partial w_{j}} \alpha+\frac{\partial g_{n}}{\partial w_{i}} \frac{\partial \alpha}{\partial w_{j}}, \ldots\right)
\end{array}
$$


And the $2 n$-th column is

$$
\begin{array}{r}
\mathbf{a}_{2 n}=\left(0, \ldots, 0, \ldots, 0, \Delta_{\bar{F}}, \ldots, \frac{\partial^{2} h}{\partial w_{n+i} \partial w_{2 n}}-\frac{\partial^{2} g_{n}}{\partial w_{n+i} \partial w_{2 n}} \alpha-\frac{\partial g_{n}}{\partial w_{n+i}} \frac{\partial \alpha}{\partial w_{2 n}}\right. \\
\left.\ldots, \frac{\partial \alpha}{\partial w_{2 n}}, \ldots,-\frac{\partial^{2} h}{\partial w_{i} \partial w_{2 n}}+\frac{\partial^{2} g_{n}}{\partial w_{i} \partial w_{2 n}} \alpha+\frac{\partial g_{n}}{\partial w_{i}} \frac{\partial \alpha}{\partial w_{2 n}}, \ldots\right) .
\end{array}
$$

Since $F$ is isotropic, for $j \leq n$, we have

$$
\begin{aligned}
0 & =\dot{\omega}\left(\mathbf{a}_{j}, \mathbf{a}_{2 n}\right) \\
& =\frac{\partial^{2} h}{\partial w_{j} \partial w_{2 n}}-\frac{\partial^{2} g_{n}}{\partial w_{j} \partial w_{2 n}} \alpha-\frac{\partial g_{n}}{\partial w_{j}} \frac{\partial \alpha}{\partial w_{2 n}}+\frac{\partial g_{n}}{\partial w_{j}} \frac{\partial \alpha}{\partial w_{2 n}}-\Delta_{\bar{F}} \frac{\partial \alpha}{\partial w_{j}} \\
& =\frac{\partial^{2} h}{\partial w_{j} \partial w_{2 n}}-\frac{\partial^{2} g_{n}}{\partial w_{j} \partial w_{2 n}} \alpha-\Delta_{\bar{F}} \frac{\partial \alpha}{\partial w_{j}},
\end{aligned}
$$

and

$$
\begin{aligned}
0 & =\dot{\omega}\left(\mathbf{a}_{n+j}, \mathbf{a}_{2 n}\right) \\
& =\frac{\partial^{2} h}{\partial w_{n+j} \partial w_{2 n}}-\frac{\partial^{2} g_{n}}{\partial w_{n+j} \partial w_{2 n}} \alpha-\frac{\partial g_{n}}{\partial w_{n+j}} \frac{\partial \alpha}{\partial w_{2 n}}+\frac{\partial g_{n}}{\partial w_{n+j}} \frac{\partial \alpha}{\partial w_{2 n}}-\Delta_{\bar{F}} \frac{\partial \alpha}{\partial w_{n+j}} \\
& =\frac{\partial^{2} h}{\partial w_{n+j} \partial w_{2 n}}-\frac{\partial^{2} g_{n}}{\partial w_{n+j} \partial w_{2 n}} \alpha-\Delta_{\bar{F}} \frac{\partial \alpha}{\partial w_{n+j}} .
\end{aligned}
$$

Thus in both cases, we have

$$
0=\dot{\omega}\left(\mathbf{a}_{j}, \mathbf{a}_{2 n}\right)=\frac{\partial^{2} h}{\partial w_{j} \partial w_{2 n}}-\frac{\partial^{2} g_{n}}{\partial w_{j} \partial w_{2 n}} \alpha-\Delta_{\bar{F}} \frac{\partial \alpha}{\partial w_{j}} .
$$

Since $\Delta_{\bar{F}}(u, v)=0$, we have

$$
\frac{\partial^{2} h}{\partial w_{n+j} \partial w_{2 n}}(u, v)-\frac{\partial^{2} g_{n}}{\partial w_{n+j} \partial w_{2 n}}(u, v) \alpha(u, v)=0 .
$$

Now, since we assumed that $\frac{\partial \alpha}{\partial w_{2 n}}(u, v)=0$, from (3.2) and (3.4), for all $i$, we have

$$
\frac{\partial \dot{f}_{i}}{\partial w_{2 n}}(u, v)=0, \quad \frac{\partial \dot{g}_{i}}{\partial w_{2 n}}(u, v)=0 .
$$

Thus $J(F)(u, v)$ has corank 1 .

We can write

$$
(j F)^{-1}\left(I_{1}\right)=\left\{(u, v) \in U: \Delta_{\bar{F}}(u, v)=0 \quad \text { and } \quad \frac{\partial \alpha}{\partial w_{2 n}}(u, v)=0\right\}
$$

and by Proposition 3.1 we have

Proposition 3.2. Let $F:(U, 0) \rightarrow T \mathbb{R}^{2 n}$ be an isotropic map-germ generated by a smooth function-germ $h$ satisfying (3.1) such that corank of JF $(0,0)$ is equal to 1 . Then $j^{1} F:(U, 0) \rightarrow I(4 n, 2 n)$ is transversal to $I_{1}$ at $(0,0)$ if and only if

$$
\operatorname{rank} J\left(\Delta_{\bar{F}}, \frac{\partial \alpha}{\partial v_{n}}\right)(0,0)=2
$$

Now we compare a generic property of a smooth Lagrangian submanifold $L \subset T \mathbb{R}^{2 n}$ generated by a versal Morse family germ with a corresponding one obtained for an 
isotropic mapping $F$. Let $\left.\pi\right|_{L}: L \rightarrow \mathbb{R}^{2 n}$ denote the restriction of $\pi: T \mathbb{R}^{2 n} \rightarrow \mathbb{R}^{2 n}$ to $L$ and

$$
\Sigma^{i}\left(\left.\pi\right|_{L}\right)=\left\{p \in L: \operatorname{corank} d\left(\left.\pi\right|_{L}\right)_{p}=i\right\}
$$

It is well known that

1) the codimension of $\Sigma^{1}\left(\left.\pi\right|_{L}\right)$ in $L$ is 1 and

2) the codimension of $\Sigma^{i}\left(\left.\pi\right|_{L}\right)$ in $L$ is $i(i+1) / 2 \geq 3$ if $i \geq 2$.

On the other hand for a map-germ $\bar{F}:(U, 0) \rightarrow \mathbb{R}^{2 n}$ such that $j^{1} \bar{F}$ is transversal to the corank $k$ stratum in the jet space for all $k=0, \ldots, 2 n$ we have a corresponding property,

1) the codimension of $\Sigma^{1}(\bar{F})$ in $U$ is 1 and

2) the codimension of $\Sigma^{i}(\bar{F})$ in $U$ is $i^{2} \geq 4$ if $i \geq 2$.

Let us denote

$$
\Sigma^{k}\left(\mathbb{R}^{2 n}, \mathbb{R}^{2 n}\right)=\left\{\sigma \in J^{1}\left(\mathbb{R}^{2 n}, \mathbb{R}^{2 n}\right): \operatorname{corank} \sigma=k\right\}
$$

Then we have

Lemma 3.3. Let $L \subset T \mathbb{R}^{2 n}$ be a Lagrangian submanifold. Let $p \in L$ and suppose that the corank of the differential $d\left(\left.\pi\right|_{L}\right)_{p}: T_{p} L \rightarrow T_{\pi(p)} \mathbb{R}^{2 n}$ is $k \geq 2$. Then $j^{1}\left(\left.\pi\right|_{L}\right): L \rightarrow$ $J^{1}\left(L, \mathbb{R}^{2 n}\right)$ is not transversal to $\Sigma^{k}\left(L, \mathbb{R}^{2 n}\right) \subset J^{1}\left(L, \mathbb{R}^{2 n}\right)$ at $p$.

And using Lemma 3.3 we get

TheOREM 3.4. Suppose that $n \geq 2$ and $k \geq 2$. Let $\bar{F}:(U, 0) \rightarrow \mathbb{R}^{2 n}$ be a smooth map-germ such that $j^{1} \bar{F}(0,0) \in \Sigma^{k}$ and that $j^{1} \bar{F}$ is transversal to $\Sigma^{k}\left(\mathbb{R}^{2 n}, \mathbb{R}^{2 n}\right)$. Let $F:(U, 0) \rightarrow T \mathbb{R}^{2 n}$ be a corank 1 isotropic map-germ along $\bar{F}$. Then $F$ is neither a Lagrangian immersion nor a Lagrangian stable isotropic map-germ.

In [8] G. Ishikawa classified Lagrangian stable isotropic map-germs of corank 1 and named them open Whitney umbrellas (cf. [7, 12]). In our context, his theorem can be stated as follows.

TheOREM 3.5 (Ishikawa [8]). Let $F:(U, 0) \rightarrow T \mathbb{R}^{2 n}$ be a Lagrangian stable isotropic map-germ of corank 1 . Then $F$ is Lagrangian equivalent to one of the following normal forms $F_{2 n, k}=(f, g, \dot{f}, \dot{g}):(U, 0) \rightarrow T \mathbb{R}^{2 n}$ defined by

$$
\begin{aligned}
f_{i}(u, v) & =u_{i}=w_{i}, \\
g_{i}(u, v) & =v_{i}=w_{n+i}, \quad i=1, \ldots, n-1, \\
g_{n}(u, v) & =\frac{v_{n}^{k+1}}{(k+1) !}+u_{1} \frac{v_{n}^{k-1}}{(k-1) !}+\cdots+u_{k-1} v_{n}, \\
\dot{g}_{n}(u, v) & =w_{k} \frac{v_{n}^{k}}{k !}+\cdots+w_{2 k-1} v_{n},
\end{aligned}
$$




$$
\begin{aligned}
& \dot{f}_{i}(u, v)=\frac{-1}{i !}\left(\frac{w_{2 n}^{k+i+1}}{(k+i+1) k !}+\frac{w_{1} w_{2 n}^{k+i-1}}{(k+i-1)(k-2) !}+\cdots+\frac{w_{k-1} w_{2 n}^{i+1}}{i+1}\right) \\
& \quad \text { for } i \text { with } k \leq n+i \leq 2 k-1,
\end{aligned}
$$

where $\left(w_{1}, \ldots, w_{2 n}\right)=\left(u_{1}, \ldots, u_{n}, v_{1}, \ldots, v_{n}\right)$.

Proof of Theorem 3.4. First we show that if $j^{1} \bar{F}(0,0) \in \Sigma^{k}$ and $j^{1} \bar{F}: U \rightarrow J^{1}\left(\mathbb{R}^{2 n}, \mathbb{R}^{2 n}\right)$ is transversal to $\Sigma^{k}$ at the origin $(0,0) \in \mathbb{R}^{2 n}$ and $F:(U, 0) \rightarrow T \mathbb{R}^{2 n}$ is an isotropic mapgerm such that $\pi \circ F=\bar{F}$, then the origin $(0,0)$ is a singular point of $F$. Indeed, suppose that the origin $(0,0)$ is a regular point of $F$. Then $F:(U, 0) \rightarrow T \mathbb{R}^{2 n}$ is a Lagrangian immersion-germ, choosing $U$ small enough if necessary. Set $L=F(U)$. Then $L$ is a Lagrangian submanifold of $T \mathbb{R}^{2 n}$. We see that $\pi \circ F=\bar{F}$ and $j^{1}\left(\left.\pi\right|_{L}\right): L \rightarrow J^{1}\left(L, \mathbb{R}^{2 n}\right)$ is transversal to $\Sigma^{k} \subset J^{1}\left(L, \mathbb{R}^{2 n}\right)$ at $F(0,0)$ if and only if $j^{1} \bar{F}: U \rightarrow J^{1}\left(U, \mathbb{R}^{2 n}\right)$ is transversal to $\Sigma^{k} \subset J^{1}\left(U, \mathbb{R}^{2 n}\right)$ at $(0,0)$. But from Lemma 3.3 we know that $j^{1}\left(\left.\pi\right|_{L}\right)$ : $L \rightarrow J^{1}\left(L, \mathbb{R}^{2 n}\right)$ is never transversal to $\Sigma^{k} \subset J^{1}\left(L, \mathbb{R}^{2 n}\right)$ at $F(0,0)$. So we have got a contradiction, thus the origin is a singular point of $F$ and $F$ is not a Lagrangian immersion.

The fact that $F$ is not a Lagrangian stable isotropic map-germ of corank 1 can be seen as follows. For some symplectomorphism $\Psi: T \mathbb{R}^{2 n} \rightarrow T \mathbb{R}^{2 n}$, the composed map $\pi \circ \Psi \circ F_{2 n, \ell}$ of $\pi, \Psi$ and an open Whitney umbrella $F_{2 n, \ell}$ may have corank $k$ singular points. However, from Ishikawa's normal forms, it is easy to see that for none of them, $j^{1} \pi \circ \Psi \circ F_{2 n, \ell}$ is transversal to $\Sigma^{k}\left(\mathbb{R}^{2 n}, \mathbb{R}^{2 n}\right)$. Therefore the isotropic map-germ $F$ in question is not symplectomorphic to any of Ishikawa's normal forms $F_{2 n, \ell}$ and $F$ is not Lagrangian stable.

REMARK 3.6. For $k=1$, if the corank of the differential $d\left(\left.\pi\right|_{L}\right)_{p}: T_{p} L \rightarrow T_{\pi(p)} \mathbb{R}^{2 n}$ at $p \in L$ is 1 and $L$ is generated by a versal Morse family, then $j^{1}\left(\left.\pi\right|_{L}\right): L \rightarrow J^{1}\left(L, \mathbb{R}^{2 n}\right)$ is transversal to $\Sigma^{1} \subset J^{1}\left(L, \mathbb{R}^{2 n}\right)$ at $p$.

Now we find the condition for transversality to the corank 1 stratum of isotropic maps with corank of $J(\bar{F})$ greater than or equal to 2 .

As in Section 2, we consider an isotropic map-germ $F=(f, g, \dot{f}, \dot{g}):(U, 0) \rightarrow T \mathbb{R}^{2 n}$ with corank $J(\bar{F})(0,0)=k \geq 2$ with $\bar{F}=(f, g)$ of the form $(2.12)-(2.15)$. Let $h$ be a generating function of $F$, i.e. it satisfies (2.16). Let 


$$
\frac{\widetilde{\partial h}}{\partial v_{i}} \in\left\langle\operatorname{det} D_{2}\right\rangle=\langle\operatorname{det} J(f, g)\rangle=\left\langle\Delta_{\bar{F}}\right\rangle, \quad n-k<i \leq n,
$$

be the functions given by $(2.17)$. Since $(\dot{f}, \dot{g})$ is given by $(2.20)-(2.22), j^{1} F$ meets the codim1 stratum if and only if

$$
\operatorname{rank} \frac{\partial\left(\frac{\partial h}{\partial u_{1}}, \ldots, \frac{\partial h}{\partial u_{n}}, \frac{\partial h}{\partial v_{1}}, \ldots, \frac{\partial h}{\partial v_{n-k}}, \widetilde{\partial h} / \partial v_{n-k+1}, \ldots, \widetilde{\partial h} / \partial v_{n}\right)}{\partial\left(v_{n-k+1}, \ldots, v_{n}\right)}=k-1 .
$$

Thus we have

TheOREM 3.7. Let $F, h, \widetilde{\partial h} / \partial v_{i}, n-k<i \leq n$ be as above:

$\frac{\widetilde{\partial h}}{\partial v_{i}} \in\left\langle\Delta_{\bar{F}}\right\rangle, n-k<i \leq n \quad$ and $\quad\left(\begin{array}{c}\dot{f} \\ \dot{g}\end{array}\right)=\left(\begin{array}{cc}O & I_{n} \\ -I_{n} & O\end{array}\right)^{t}\left(\begin{array}{cc}\frac{\partial f}{\partial u} & \frac{\partial f}{\partial v} \\ \frac{\partial g}{\partial u} & \frac{\partial g}{\partial v}\end{array}\right)^{-1}\left(\begin{array}{c}\frac{\partial h}{\partial u} \\ \frac{\partial h}{\partial v}\end{array}\right)$.

1) The corank of $j^{1} F$ at $(0,0)$ is 1 if and only if

$$
\operatorname{rank}\left(\begin{array}{ccc}
\frac{\partial^{2} h}{\partial u_{1} \partial v_{n-k+1}} & \cdots & \frac{\partial^{2} h}{\partial u_{1} \partial v_{n}} \\
\cdots & \cdots & \cdots \\
\frac{\partial^{2} h}{\partial u_{n} \partial v_{n-k+1}} & \cdots & \frac{\partial^{2} h}{\partial u_{n} \partial v_{n}} \\
\frac{\partial^{2} h}{\partial v_{1} \partial v_{n-k+1}} & \cdots & \frac{\partial^{2} h}{\partial v_{1} \partial v_{n}} \\
\cdots & \cdots & \cdots \\
\frac{\partial^{2} h}{\partial v_{n-k} \partial v_{n-k+1}} & \cdots & \frac{\partial^{2} h}{\partial v_{n-k} \partial v_{n}} \\
\frac{\partial^{2} h}{\partial v_{n-k+1} \partial v_{n-k+1}} & \cdots & \frac{\partial^{2} h}{\partial v_{n-k+1} \partial v_{n}} \\
\frac{\cdots}{\partial^{2} h} & \cdots & \frac{\cdots}{\partial^{2} h} \\
\frac{\partial v_{n} \partial v_{n-k+1}}{\partial v_{n} \partial v_{n}}
\end{array}\right)(0,0)=k-1 .
$$

2) The jet extension $j^{1} F: U \rightarrow J_{I}^{1}\left(\mathbb{R}^{2 n}, T \mathbb{R}^{2 n}\right)$ is transversal to the corank 1 stratum if and only if

$$
\operatorname{rank} J(k \times k \text { minors of the matrix }(3.6))=2 \text {, }
$$

where $J(k \times k$ minors of the matrix(3.6)) is the Jacobian matrix at $(0,0)$ of the $k \times k$ minors of the matrix (3.6) with respect to the variables $u_{1}, \ldots, u_{n}, v_{1}, \ldots, v_{n}$.

Proof. 1) follows from (3.5). 2) is also straightforward by the fact that the corank 1 stratum is defined by the minors of the matrix (3.6) and the codimension of the corank 1 stratum is 2 .

\section{References}

[1] J. Basto-Goncalves, Implicit Hamiltonian equations, Mat. Contemp. 12 (1997), 123-137.

[2] P. A. M. Dirac, Generalized Hamiltonian Dynamics, Canadian J. Math. 2 (1950), 129-148.

[3] I. Ekeland and H. Hofer, Symplectic topology and Hamiltonian dynamics, Math. Zeitschrift 200 (1989), 355-378.

[4] T. Fukuda, Local topological properties of differentiable mappings I, Invent. Math. 65 (1981), 227-250. 
[5] T. Fukuda and S. Janeczko, Singularities of implicit differential systems and their integrability, Banach Center Publications 65 (2004), 23-47.

[6] T. Fukuda and S. Janeczko, Global properties of integrable implicit Hamiltonian systems, in: Proc. of the 2005 Marseille Singularity Theory School and Conference, World Scientific, 2007, 593-611.

[7] A. B. Givental, Lagrangian embeddings of surfaces and unfolded Whitney umbrella, Funktsional. Anal. i Prilozhen. 20 (1986), 35-41 (in Russian).

[8] G. Ishikawa, Symplectic and Lagrange stabilities of open Whitney umbrellas, Invent. Math. 126, (1996), 215-234.

[9] G. Ishikawa, Determinacy, transversality and Lagrange stability, Banach Center Publ. 50 (1999), 123-135.

[10] S. Janeczko, On implicit Lagrangian differential systems, Annales Polonici Mathematici 74 (2000), 133-141.

[11] S. Janeczko and F. Pelletier, Singularities of implicit differential systems and Maximum Principle, Banach Center Publ. 62 (2004), 117-132.

[12] J. Martinet, Singularities of Smooth Functions and Maps, Cambridge Univ. Press, Cambridge, 1982.

[13] J. N. Mather, Solutions of generic linear equations, in: Dynamical Systems, 1972, 185-193.

[14] B. Morin, Formes canoniques des singularités d'une application différentiable, Comptes Rendus de l'Académie des Sciences 260 (1965), 5662-5665, 6503-6506.

[15] R. Thom, Sur les équations différentielles multiformes et leurs intégrales singulières, Colloque E. Cartan, Paris, 1971.

[16] A. Weinstein, Lectures on Symplectic Manifolds, CBMS Regional Conf. Ser. in Math. 29, AMS, Providence, RI, 1977. 
